\section{OC-006 SETTING UP A CENTRALISED DECONTAMINATION SERVICE: LESSONS LEARNT}

doi:10.1136/gutjnl-2012-302514a.6

V Jackson, J Mitchell, D Jones.* Department of Endoscopy, Aintree Hospitals NHS Foundation Trust, Liverpool, UK

Introduction Decontamination is moving towards a centralised service within secondary care. The aim of such is to maximise expertise and maintain quality standards. The development of centralised decontamination will minimise risk and assist the audit process. Within the authors workplace the decision was taken to house this service within the endoscopy department.

Methods An analysis of current services that used flexible endoscopes was undertaken including workforce, equipment and current usage of decontamination within the area it was undertaken. This included endoscopy, ENT \& MFU outpatient and inpatient, ITU, Theatres. This led to a business case to request appropriate resource to enable implementation and deliver of required service for all users. Results There was a difference in perceptions of a centralised decontamination service from all stakeholders. Each department had a tunnel vision with little regard for others. This led to a conflict due to all users having different priorities with little understanding of other pressures. Out of hours decontamination was an expectation from management with no acknowledgement of the impact this would have on the workforce. It was understood that all capital equipment would be pooled, however this was not accepted by some departments and led to shortages of endoscopes as services were reluctant to "share". The development of a band 4 decontamination supervisor helped the implementation of the service by offering expertise and leadership to all user groups. Management expectations of the service were often unrealistic due to lack of expertise in the area. The band 4 role offered a realistic solution using the knowledge and skills from their own experience in the field. Savings were delivered by the reduction of reprocessors required for maintenance contracts.

Conclusion Clear understanding of objectives is required prior to the development of any service. It is vital that a stakeholders group is formed and gives all involved the time to voice any concerns at any stage. Workforce requirements must be analysed to ensure the right skill mix is available to deliver the required knowledge and skills for implementation and sustainability. There needs to be greater emphasis on business continuity plans when cenralising any service.

Competing interests None declared.

\section{BSG plenary session}

\section{OC-007 THE NATIONAL COLONOSCOPY AUDIT: A NATIONWIDE ASSESSMENT OF THE QUALITY AND SAFETY OF COLONOSCOPY IN THE UK}

doi:10.1136/gutjnl-2012-302514a.7

${ }^{1} \mathrm{D}$ Gavin, ${ }^{2} \mathrm{R}$ Valori, ${ }^{1} \mathrm{~J}$ Anderson, ${ }^{3} \mathrm{M}$ Donnelly, ${ }^{4} \mathrm{~J} \mathrm{G}$ Williams, ${ }^{5} \mathrm{E}$ Swarbrick. ${ }^{1}$ Department of Gastroenterology, Gloucestershire Hospitals NHS Foundation Trust, Cheltenham, UK; ${ }^{2}$ Department of Gastroenterology, Gloucestershire Hospitals NHS Foundation Trust, Gloucester, UK; ${ }^{3}$ Department of Gastroenterology, Sheffield Teaching Hospitals NHS Foundation Trust, Sheffield, UK; ${ }^{4}$ Department of General Surgery, Royal Wolverhampton Hospitals NHS Foundation Trust, Wolverhampton, UK; ${ }^{5}$ Department of Gastroenterology, Royal Wolverhampton Hospitals NHS Foundation Trust, Wolverhampton, UK

Introduction Colonoscopy is the gold standard procedure for the diagnosis and non-surgical management of colonic disease. Poor quality colonoscopy is associated with complications and reduced effectiveness in disease prevention. A previous large-scale study of colonoscopy practice in the UK demonstrated disappointing results with poor caecal intubation rates and higher than expected complication rates. ${ }^{1}$ Since then there has been significant investment in endoscopic training, a quality assurance framework for endoscopy units has been implemented and the National Bowel Cancer Screening Program has been rolled out nationally. The aim of this study was to assess the quality of contemporary UK colonoscopy.

Methods A nationwide audit of colonoscopy practice was conducted over a 2-week period from 28 February 2011 until 11 March 2011. The study was performed prospectively, with data entry occurring electronically through a purpose built website (http://www. endoaudit.com). All units performing $>100$ colonoscopies annually on NHS patients were included. All colonoscopies performed on adults ( $>16$ years of age) were included. Data on key performance indicators and complications was collected and analysed.

Results Data on 20085 colonoscopies and 2681 colonoscopists were collected from 301 units. A validation exercise indicated that data were collected on $>94 \%$ of all procedures performed nationally. The overall, unadjusted caecal intubation rate (CIR) was $92.3 \%$. When adjusted for impassable strictures and poor bowel preparation the CIR was $95.8 \%$. The polyp detection rate (PDR) was $32.1 \%$. The PDR for significant polyps (>1 cm diameter) was $11.7 \%$. $92.3 \%$ of resected polyps were retrieved. $90.2 \%$ of procedures achieved acceptable levels of patient comfort. A total of eight perforations and 52 significant haemorrhages were reported. Eight patients underwent surgery as a consequence of a complication.

Conclusion This is the first national audit of colonoscopy that has successfully captured the majority of adult colonoscopy performed during a defined time period. The data confirm that there has been a significant improvement in performance of colonoscopy in the UK since the last study reported 7 years ago (CIR 76.9\%) and that performance is above the required national standards.

Competing interests None declared.

\section{REFERENCE}

1. Bowles CJ, Leicester R, Romaya C, et al. A prospective study of colonoscopy practice in the UK today: are we adequately prepared for national colorectal cancer screening tomorrow? Gut 2004;53:277-83.

\section{OC-008 DEFINING THE GENETIC LANDSCAPE OF OESOPHAGEAL ADENOCARCINOMA BY NEXT-GENERATION SEOUENCING}

doi:10.1136/gutjnl-2012-302514a.8

${ }^{1} \mathrm{~J} \mathrm{M} J$ Weaver, ${ }^{* 2} \mathrm{~N}$ B Shannon, ${ }^{2} \mathrm{M}$ Smith, ${ }^{2} \mathrm{M}$ Dunning, ${ }^{1} \mathrm{C}$ A Ong, ${ }^{1} \mathrm{C}$ Ross-Innes, ${ }^{3} \mathrm{~T}$ Underwood, ${ }^{2} \mathrm{~A}$ Lynch, ${ }^{2} \mathrm{M}$ Eldridge, ${ }^{2} \mathrm{C}$ Caldas, ${ }^{1} \mathrm{P}$ Edwards, ${ }^{2} \mathrm{~S}$ Tavare, ${ }^{1} \mathrm{R}$ C Fitzgerald. ${ }^{1}$ Hutchison-MRC, UK; ${ }^{2} \mathrm{CRI}$, Cambridge, UK; ${ }^{3}$ Southampton Hospital, Southampton, UK

Introduction Despite its dismal prognosis and steady increase in prevalence little is known about the genetic alterations that drive oesophageal adenocarcinoma. As part of the International Cancer Genome Consortium: Oesophageal Adenocarcinoma (ICGC-OAC) we performed a pilot study, sequencing the genomes of $32 \mathrm{OAC}$ samples, to assess the feasibility of initiating a large scale project sequencing a total of 500 OAC genomes.

Methods A total of 56 genomes were selected for sequencing including 32 OAC genomes-16 chemotherapy-Naive, 16 chemotherapy-treated-and 24 matched normal genomes. Whole genome sequencing was performed on the Illumina Hiseq 2000 platform. Initial bioinformatic analysis, run by Illumina using the Casava pipeline, detected single nucleotide variants (SNVs), small ( $<50 \mathrm{bp})$ Insertion and Deletions events (INDELs) and large scale structural variants (SVs). Additionally, bioinformatic analysis of SVs was performed using a custom Perl script. To determine the specificity of 
the bioinformatic approach a subset of SNVs and SVs were selected for verification by Sanger capillary sequencing and PCR respectively. Results A minimum of 50-fold mappable sequence data were generated for each of the 56 genomes. 161/167 (96\%) of predicted SNVs were confirmed as somatic, two were miscalled germline variants while four were undetectable in either sample. For 2/75 (3\%) SVs PCR amplicons could not be generated, for 18 of 75 SVs (24\%) a PCR amplicon was detectable in the normal showing them to be germline polymorphisms. The True positive rate for SV detection was therefore $73 \%$. Comparison of SNV information across all 24 samples revealed many recurrently mutated genes. These include previously reported mutations in TP53, CDKN2A and APC among others. No genes were significantly associated with chemotherapy-treated or chemotherapy-naive samples.

Conclusion Analysis of the Illumina bioinformatic pipeline suggests it is highly specific ( $96 \%$ true positive rate) for somatic SNVs. A true positive rate of $73 \%$ for SV detection is comparable to recent literature. Further analysis to determine the sensitivity of this pipeline is ongoing including resequencing of putatively non-mutated genes in samples sent for WGS and the application of alternative bioinformatic approaches for the calling of SNVs, INDELs and SVs. Initial analysis of the SNV data from 32 tumour genomes has revealed several recurrently mutated genes known to be altered in OAC validating the ability of our approach to detect candidate "driver" genes.

Competing interests None declared.

\section{DDF inflammatory bowel disease symposium OC-009 HUMAN ANTI-MICROBIAL V $\delta 2+$ T-CELLS ARE NOVEL INTESTINAL LYMPHOCYTES WITH FUNCTIONAL RELEVANCE IN CROHN'S DISEASE}

\section{doi:10.1136/gutjnl-2012-302514a.9}

${ }^{1} \mathrm{~N}$ E McCarthy, ${ }^{*} \mathrm{C}$ R Hedin, ${ }^{1} \mathrm{~A}$ Vossenkämper, ${ }^{1} \mathrm{~S}$ Bhattacharjee, ${ }^{1} \mathrm{~S}$ G Brown, ${ }^{1} \mathrm{~T}$ J Sanders, ${ }^{1} \mathrm{E} M \mathrm{M}$ Giles, ${ }^{2} \mathrm{~K}$ Whelan, ${ }^{3} \mathrm{~J} 0$ Lindsay, ${ }^{1} \mathrm{~A} J$ Stagg. ${ }^{1}$ Centre for Immunology \& Infectious Disease, The Blizard Institute, Barts \& The London School of Medicine \& Dentistry, Queen Mary University of London, London, UK; ${ }^{2}$ Nutritional Sciences Division, King's College London, London, UK; ${ }^{3}$ Digestive Diseases Clinical Academic Unit, Barts \& The London School of Medicine \& Dentistry, London, UK

Introduction V $\gamma 9 \mathrm{~V} \delta 2+$ "unconventional" (V82) T-cells are a population of circulating anti-microbial lymphocytes found only in higher primates and whose role in human intestinal immunity is unknown. In macaques, microbe-activated V $\delta 2 \mathrm{~T}$-cells expand and accumulate in mucosal tissues, and human V82T-cells can produce key mediators of intestinal inflammation such as IFN $\gamma$, TNF $\alpha$ and IL-17A in response to bacterial species present among the gut microbiota. We therefore hypothesised that V $\delta 2 \mathrm{~T}$-cells might contribute to the pathogenesis of Crohn's disease (CD).

Methods Disaggregated intestinal biopsies and peripheral blood were analysed by flow-cytometry in CD patients $(n=22)$, and healthy controls $(n=36)$. Blood and biopsy-derived cell suspensions were stimulated with microbial phosphoantigen (HDMAPP) and IL-2 in vitro to determine V $\delta 2 \mathrm{~T}$-cell phenotype, cytokine production and proliferative potential in the presence or absence of azathioprine.

Results Blood V $\delta 2 \mathrm{~T}$-cells proliferated, expressed "gut-homing" integrin $\beta 7$, and produced IFN $\gamma$ and TNF $\alpha$ upon activation with HDMAPP and IL-2 in vitro. V $\delta 2 \mathrm{~T}$-cells were also identified by confocal microscopy in both healthy and inflamed colonic lamina propria. In contrast to their blood counterparts, mucosal V $\delta 2 \mathrm{~T}$-cells expressed high levels of CD103 integrin, which is implicated in interactions with the intestinal epithelium. Although the frequency of mucosal V $\delta 2 \mathrm{~T}$-cells was low, these cells proliferated rapidly and up-regulated CD70 co-stimulatory molecule upon exposure to HDMAPP and IL-2 in vitro, consistent with responsiveness to the gut microbiota. In $\mathrm{CD}$ patients receiving azathioprine therapy, V82T-cells were selectively lost from the blood and were markedly depleted from the lamina propria. Accordingly, physiological concentrations of azathioprine were sufficient to block HDMAPP activation of V82T-cells in vitro.

Conclusion Human V $\delta 2 \mathrm{~T}$-cells primarily reside in the blood but display gut-homing potential upon microbial activation and can be detected in the intestinal mucosa. Intestinal V $82 \mathrm{~T}$-cells may contribute to local pro-inflammatory responses against the gut microbiota but are depleted by azathioprine therapy in CD.

Competing interests None declared.

\section{OC-010 DETAILED ANALYSIS OF ATG16L1 DEMONSTRATES GENE-WIDE EXTENT OF ASSOCIATION WITH CROHN'S DISEASE SUSCEPTIBILITY}

doi:10.1136/gutjnl-2012-302514a.10

1,2,3 J Van Limbergen, ${ }^{4} \mathrm{~B}$ Kabakchiev, ${ }^{4} \mathrm{~J}$ Stempak, ${ }^{5} \mathrm{P}$ Schumm, ${ }^{6} \mathrm{~W} \quad \mathrm{Xu}$, ${ }^{1} \mathrm{P}$ Henderson, ${ }^{2} \mathrm{~A}$ Griffiths, ${ }^{7} \mathrm{~S}$ Girardin, ${ }^{3} \mathrm{D}$ Philpott, ${ }^{4} \mathrm{M}$ Silverberg. ${ }^{1}$ Child Life and Health, University of Edinburgh, Edinburgh, UK; ${ }^{2}$ Division of Gastroenterology, Hepatology and Nutrition, Toronto sick kids, Toronto, Canada; ${ }^{3}$ Department of Immunology, University of Toronto, Toronto, Canada; ${ }^{4}$ Division of Gastroenterology, Mount Sinai Hospital, Toronto, Canada; ${ }^{5}$ Department of Health Studies, University of Chicago, Chicago, USA; ${ }^{6}$ Dalla Lana School of Public Health, University of Toronto, Toronto, Canada; ' Laboratory Medicine and Pathobiology, University of Toronto, Toronto, Canada

Introduction ATG16L1 has been implicated in the susceptibility to Crohn's disease (CD), notably the T300A (rs2241880, exon 9). In ATG16L1-deficient and hypomorphic mice, autophagy, Paneth-cell homeostasis and IL-1b secretion were dependent on ATG16L1 (Saitoh et al Nature 2008, Cadwell et al Cell 2010). In contrast, studies focusing on T300A have shown conflicting results (Kuballa et al PLoS ONE 2008, Fujita et al JBC 2009). The association at other susceptibility loci (eg, NOD2 and IL23R) consists of common and rare variants (Rivas et al Nat Genet 2011). Exon-sequencing studies have not demonstrated rare variants within the 18 exons of ATG16L1. Our aim was to analyse the ATG16L1 association signal, specifically focusing on common variants.

Methods 38 single nucleotide polymorphisms (SNPs) spanning the ATG16L1 gene were imputed for 1735 subjects (800 CD/935 controls) (Franke et al Nat Genet 2010). Single SNP and haplotype frequency ( $>1 \%$, solid spine of Linkage disequilibrium (LD), $\left.\mathrm{D}^{\prime}>0.8\right)$ association and permutation $(\mathrm{n}=10000)$ analyses were performed (Haploview). Logistic regression (using SNPs $p<0.05$ on permutation analysis) was performed (SPSS). Monte Carlo simulation $(n=10000, R)$ was used to assess the difference in the number of haplotype blocks, based on $\mathrm{D}^{\prime}>0.8$, between $\mathrm{CD}$ and controls.

Results Single SNP permutation analysis yielded association of 16 markers $(p<0.001)$, from intron 1 (rs6752107) to the 3'UTR (rs1045100). Analysis of $D^{\prime}$ and $r^{2}$ characteristics, showed 12 SNPs were in complete LD with rs2241880 ( $D^{\prime}$ and $r^{2} 0.98-1$ ). rs6758317 $\left(r^{2}=0.25\right.$, intron 2), rs3792106 $\left(r^{2}=0.76\right.$, intron 11), rs4663396 $\left(r^{2}=0.25\right.$, intron 12) and $r s 1045100\left(r^{2}=0.84\right)$ demonstrated strong association, independent of rs2241880. Regression analysis retained rs3792106, rs6754677 (intron 14) and rs1045100 (3'UTR) ( $p<0.05)$. A Monte Carlo simulation showed no significant difference in the number of haplotype blocks between CD and controls $(p=1)$. A strong association with CD was shown for the haplotype block containing the rs2241880 and for the block containing rs1045100, both $p<10^{-5}$ Conclusion We demonstrated that the rs2241880 alone is not sufficient to explain the strong ATG16L1 association. Additional variants, independent of rs2241880, could implicate any of the coiled-coil domain, the WD domain and/or the 3' UTR, in CD susceptibility.

Competing interests None declared. 